\title{
Paclitaxel-loaded micro or nano transfersome formulation into novel tablets for pulmonary drug delivery via nebulization
}

\author{
Iftikhar Khan $^{\mathrm{a}, *}$, Maria Apostolou ${ }^{\mathrm{a}}$, Ruba Bnyan ${ }^{\mathrm{a}}$, Chahinez Houacine ${ }^{\mathrm{b}}$, Abdelbary Elhissi ${ }^{\mathrm{c}}$, \\ Sakib S Yousaf ${ }^{b, *}$ \\ ${ }^{a}$ School of Pharmacy and Biomolecular Sciences, Liverpool John Moores University, Liverpool L3 3AF, United Kingdom \\ ${ }^{\mathrm{b}}$ School of Pharmacy and Biomedical Sciences, University of Central Lancashire, Preston PR1 2HE, United Kingdom \\ ${ }^{c}$ Pharmaceutical Sciences Section, College of Pharmacy, Qatar University, P.O. Box 2713, Doha, Qatar
}

\section{A R T I C L E I N F O}

\section{Keywords:}

Paclitaxel

Protransfersomes

Transfersomes

Nebulization

Tablets

\begin{abstract}
A B S T R A C T
A simplistic approach was adopted to manufacture novel paclitaxel (PTX) loaded protransfersome tablet formulations for pulmonary drug delivery. The large surface area offered by the pulmonary system acts as a desirable site for anti-cancer drug deposition; offering localized effect within the lungs. Protransfersomes are dry powder formulations, whereas transfersomes are liquid dispersions containing vesicles generated from protransfersomes upon hydration. Protransfersome powder formulations (F1-F27) (referred as Micro formulations based on transfersomes vesicles size post hydration) were prepared by employing a phospholipid (Soya phosphatidylcholine (SPC)), three different carbohydrate carriers (Lactose monohydrate, LMH; Microcrystalline cellulose, MCC; and Starch), three surfactants (i.e. Span 80, Span 20 and Tween 80) in three different lipid phase to carrier ratios (i.e. 1:05, 1:15 and 1:25 w/w), with the incorporation of PTX as a model drug. Hydrophobic chain of SPC may enhance PTX solubility, entrapment and targetted delivery via transfersome vesicles. Out of the 27 Micro protransfersome formulations, PTX-loaded LMH powder formulations F3, F6 and F9 (i.e. 1:25 w/w lipid phase to carrier ratio) exhibited excellent powder flowability via angle of repose (AOR) and good compressibility index due to associated smaller and uniform particle size and shape of LMH. Following hydration, these formulations also showed smaller volume median diameters (VMD) in micrometres (5.65 $\pm 0.85-6.76 \pm 0.61 \mu \mathrm{m})$ and PTX entrapment of 93-96\%. Hydrated transfersome formulations (F3, F6 and F9) were converted into Nano size via probe sonication and referred to as Nano formulations. These Nano formulations were converted into dry powder via spray drying (SD) (F3NSD, F6NSD and F9NSD) or freeze drying (FD) (F3NFD, F6NFD and F9NFD). Post manufacture of protransfersome tablets (i.e. 9 formulations), quality control tests were conducted in accordance to British Pharmacopeia (BP). Only the Micro formulations protransfersome tablets (i.e. F3, F6 and F9) passed the uniformity of weight test, exhibited high crushing strength and tablet thickness when compared to SD or FD protransfersome tablets. Micro protransfersome formulations (i.e. F3, F6 and F9) into tablets demonstrated a shorter nebulization time and high output rate when using Ultrasonic nebulizer compared to Vibrating mesh nebulizer (i.e. Omron NE U22). Based on formulations, characterizations and nebulizer performance; Micro protransfersome tablet formulations F3, F6 and F9 (i.e. 1:25 $\mathrm{w} / \mathrm{w})$ and Ultrasonic nebulizer were found to be a superior combination, eliciting enhanced output efficiency. Moreover, PTX-loaded F3, F6 and F9 tablet formulations (10\%) exhibited toxicity (60, 68 and $67 \%$ cell viability) to cancer MRC-5 SV2 (i.e. immortalized human lung cells) while safe to MRC-5 (normal lung fibroblast cells) cell lines.
\end{abstract}

\section{Introduction}

PTX is one of the most widely investigated anti-cancer agents; possessing high anti-tumour activity against lung, breast, neck, head and ovarian cancers, as well as Kaposi's sarcoma (McGuire et al., 1989;
Spencer and Faulds, 1994; He et al., 2003). PTX reduces the critical concentration of microtubule proteins which are essential for microtubule assembly, a crucial aspect of cancerous cell growth (Orr et al., 2003). PTX prevents angiogenesis-related cell migration and proliferation, in addition to collagenase secretion. PTX also possesses low water

\footnotetext{
* Corresponding authors.

E-mail addresses: I.Khan@ljmu.ac.uk (I. Khan), SYousaf6@uclan.ac.uk (S.S. Yousaf).
} 
solubility; in addressing this through the addition of excipients (which aid in water solubility in intravenous formulation), incidence of side effects are notable, including; diarrhoea, nausea, vomiting, hypersensitivity reactions and neutropenia (Gelderblom et al., 2001). These unwanted reactions originating from PTX formulations can be diminished when phospholipids and surfactants are employed in combinations, in the form of transfersome formulations. These inherently improve solubility (due to the presence of hydrocarbon chains).

The term "Transfersome" is a combination of Latin and Greek words, with the derived meaning of "carrying body" (Bhushan Rajendra and Nayan Ashok, 2017). Transfersomes are also referred to as ultradeformable vesicles, possessing an aqueous core suitable for the entrapment of hydrophilic drugs. This core is surrounded by concentric bilayers of phospholipid in which hydrophobic drugs may be successfully entrapped. The phospholipid component of transfersomes is associated with stability problems (i.e. aggregation, fusion and leakage of drugs from the vesicles) (Khan et al., 2016). Dry powder formulations of transfersomes (protransfersomes) have been investigated as a means to increase the stability of the vesicle formulation. Succinctly, these dry formulations are comprised of a thin lipid film (e.g. a mixture of phospholipid, surfactant, drug and with or without cholesterol) deposited over carbohydrate carrier particles (e.g. sucrose, sorbitol or mannitol). Transfersomes are generated from protransfersomes by a simple hydration method (Subramanian et al., 2016). However, maintaining the formulation in powder form is highly challenging due to bulk associated with powder formulations, difficulty in large scale manufacture, and health risks associated with the inhalation of ultrafine particles present in powder formulations (EPA, 2013). Thus, the compression of powders into tablet form is appealing, offering number of select advantages, including; ease of large scale manufacturing, handling and packaging.

With respect to pulmonary disease states, inhalation of vesicles via nebulization enhances residence time of API's in the lungs, potentially enhancing therapeutic effect without inducing significant systemic side effects (Khan et al., 2016). Lipid-based vesicles may be deposited into artificial lung models (e.g. a Two Stage Impinger (TSI)) via Air-jet, Ultrasonic or Vibrating mesh nebulizers. Air-jet nebulizer may damage the delicate vesicles due to compressed gas passing through venture (0.3-0.7 $\mathrm{mm}$ in diameter) forming a jet of formulation liquid in the nebulizer, followed by collapsing into small droplets due to surface tension (McCallion et al., 1996; Aulton and Taylor, 2018b). Ultrasonic nebulizers produces high frequency vibrations via piezoelectric crystals, producing aerosol droplets. However, these nebulizers are associated with heat production, which may damage thermolabile compounds (Leung et al., 1996; Taylor and McCallion, 1997). Vibrating mesh nebulisers possess a perforated plate containing aperture of specific diameters in micrometres, thus aerosols of a specific size are produced and delivered (Aulton and Taylor, 2018b). However, vesicle damage is possible during aerosol formation when this nebulizer is employed (McCallion et al., 1996; O'Callaghan and Barry, 1997). It is evident that there are a mixture of benefits and pitfalls when employing nebulizers. In spite of this, the flexibility and resilience exhibited by transfersomes provides scope for investigation as nebulized formulations.

This study aims to develop and determine PTX-loaded protransfersome powder formulations via a slurry-based method, followed by compression into novel PTX-loaded protransfersome tablets. Initially, 27 protransfersome powder formulations using one phospholipid (SPC), three carbohydrate carriers (LMH, MCC and Starch), three surfactants (Span 80, Span 20 and Tween 80) and three lipid phase to carrier ratios (1:05, 1:15 and 1:25 w/w) were prepared and investigated to find the best formulation with high powder flowability. Post tablet manufacturing, quality control tests were performed according to BP standards in order to assess the quality of tablets via uniformity of weight, hardness (i.e. crushing strength), thickness and disintegration time. Selected PTX-loaded protransfersome tablets were aerosolized using two different nebulizers to generate inhalable aerosol droplets. The performance of tablet formulations and nebulizer types were characterized with the help of nebulization time, sputtering time, mass output and output rate of aerosols. The cytotoxicity of transfersome vesicles hydrated from protransfersome tablets were further investigated in MRC-5 (i.e. normal lung fibroblast cells) and MRC-5 SV2 (i.e. cancer immortalized lung cells) cell lines. This is the first study where PTX-loaded protransfersome tablets were employed for aerosolization as well as cell lines study.

\section{Materials and methods}

\subsection{Materials}

Cholesterol, Span 20, Span 80, Tween 80 and Fetal bovine serum (FBS) were purchased from Sigma-Aldrich, UK. Lactose Monohydrate (LMH), Microcrystalline Cellulose (MCC; Avicel pH 12), and Starch (Starch-150) were procured from VWR (BDH Prolab), UK. Analar grade acetonitrile, methanol, absolute ethanol, deionized water and Millipore filters (10 KDa) were obtained from Fischer Scientific, UK. Soya Phosphatidylcholine (SPC; Lipoid S-100, 94\% purity) was acquired from Lipoid, Steinhausen, Switzerland. Paclitaxel was purchased from ChemieTek, Indianapolis, USA. The Vibrating mesh nebulizer (i.e. Omron NE U22) was purchased from Omron Healthcare UK Ltd, UK. The Ultrasonic nebulizer was procured from Uniclife Healthcare LTD, UK. Dulbecco's Modified Eagle Medium (DMEM) with high glucose, TryplTrypLE ${ }^{\mathrm{TM}}$ Express Enzyme (1X), with phenol red, and AlamarBlue ${ }^{\mathrm{TM}}$ cell viability reagent were bought from Gibco, Thermofisher, UK. MRC5 (ECACC 05090501) and MRC-5 SV2 (ECACC 84100401) cell lines were obtained from Public Health England (Salisbury, UK).

\subsection{Preparation of paclitaxel loaded protransfersomes}

Protransfersomes were prepared using three different carbohydrate carriers: Lactose monohydrate (LMH), microcrystalline cellulose (MCC) or Starch via a Slurry method (Khan et al., 2015). Three surfactants were employed, based on their hydrophilic-lipophilic balance (HLB) values Span $80(\mathrm{HLB}=4.3)$, Span $20(\mathrm{HLB}=8.6)$ and Tween 80 $($ HLB $=15)$ (Bnyan et al., 2018). The formulation lipid phase $(250 \mathrm{mg}$ ) was comprised of $15 \%$ of surfactant $(37.50 \mathrm{mg})$, and $85 \%$ of the combination of SPC and cholesterol $(212.50 \mathrm{mg})$ in a 1:1 molar ratio (i.e. $141.67 \mathrm{mg}$ of SPC and $70.83 \mathrm{mg}$ of cholesterol). Paclitaxel (PTX) was employed at a $2 \mathrm{~mol} \%$ concentration to lipid phase (i.e. $7.74 \mathrm{mg}$ of PTX for Span 80, $8.10 \mathrm{mg}$ of PTX for Span 20 and $6.75 \mathrm{mg}$ of PTX for Tween 80). Moreover, three different lipid phase to carrier ratios were trialled for protransfersome formulations i.e. 1:05, 1:15 or 1:25 w/w.

In the preparation of LMH-based protransfersomes in a 1:05 w/w lipid phase to carrier ratio, LMH (1250 mg) was placed in a $100 \mathrm{ml}$ of round bottom flask (RBF). Lipid phase $(250 \mathrm{mg})$ and PTX were dissolved in absolute ethanol $(20 \mathrm{ml})$. This alcoholic solution was then poured over LMH in a RBF to make a slurry. A rotary evaporator (Buchi Rotavapor R-114, Buchi, Switzerland) equipped with a vacuum pump (Buchi Vac V-501) and water bath set at $45^{\circ} \mathrm{C}$, was used to create a negative pressure to evaporate organic solvent from the RBF; with a rotation speed of $270 \mathrm{rpm}$ for an approximately $1 \mathrm{~h}$. Following this period, negative pressure was released and the dry powder protransfersomes harvested and stored at $-18{ }^{\circ} \mathrm{C}$ for subsequent studies. This protocol/procedure was followed and repeated in order to prepare 27 protransfersome powder formulations (Table 1).

\subsection{Flow properties of protransfersome formulations}

Each coarse carbohydrate carrier (i.e. LMH, MCC and Starch) and protransfersome formulation (F1-F27) were tested for their flow properties via angle of repose (AOR) and consolidation index. The AOR method was employed using a fixed funnel method (Aulton and Taylor, 2018a), where a funnel was clamped with a stand and the lower end of 
Table 1

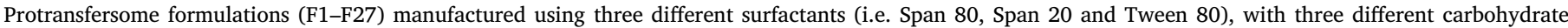

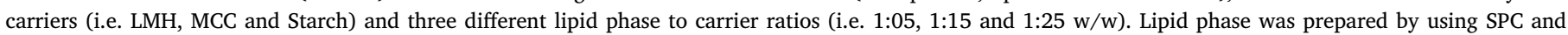
cholesterol as 1:1 $\mathrm{M}$ ratio $(85 \% \mathrm{w} / \mathrm{w})$ and surfactant $(15 \% \mathrm{w} / \mathrm{w}), \mathrm{n}=4$.

\begin{tabular}{|c|c|c|c|c|c|}
\hline Formulation Number & Phospholipid & Cholesterol & Carbohydrate Carriers & Surfactants & Lipid phase to carrier ratio $(\mathrm{w} / \mathrm{w})$ \\
\hline F1 & SPC & Cholesterol & LMH & Span 80 & 1:05 \\
\hline F2 & SPC & Cholesterol & LMH & Span 80 & $1: 15$ \\
\hline F3 & SPC & Cholesterol & LMH & Span 80 & $1: 25$ \\
\hline F4 & SPC & Cholesterol & LMH & Span 20 & 1:05 \\
\hline F5 & SPC & Cholesterol & LMH & Span 20 & $1: 15$ \\
\hline F6 & SPC & Cholesterol & LMH & Span 20 & $1: 25$ \\
\hline F7 & SPC & Cholesterol & LMH & Tween 80 & 1:05 \\
\hline F8 & SPC & Cholesterol & LMH & Tween 80 & $1: 15$ \\
\hline F9 & SPC & Cholesterol & LMH & Tween 80 & $1: 25$ \\
\hline F10 & SPC & Cholesterol & MCC & Span 80 & $1: 05$ \\
\hline F11 & SPC & Cholesterol & MCC & Span 80 & $1: 15$ \\
\hline F12 & SPC & Cholesterol & MCC & Span 80 & $1: 25$ \\
\hline F13 & SPC & Cholesterol & MCC & Span 20 & $1: 05$ \\
\hline F14 & SPC & Cholesterol & MCC & Span 20 & $1: 15$ \\
\hline F15 & SPC & Cholesterol & MCC & Span 20 & $1: 25$ \\
\hline F16 & SPC & Cholesterol & MCC & Tween 80 & 1:05 \\
\hline F17 & SPC & Cholesterol & MCC & Tween 80 & $1: 15$ \\
\hline F18 & SPC & Cholesterol & MCC & Tween 80 & $1: 25$ \\
\hline F19 & SPC & Cholesterol & Starch & Span 80 & 1:05 \\
\hline F20 & SPC & Cholesterol & Starch & Span 80 & $1: 15$ \\
\hline F21 & SPC & Cholesterol & Starch & Span 80 & $1: 25$ \\
\hline F22 & SPC & Cholesterol & Starch & Span 20 & $1: 05$ \\
\hline F23 & SPC & Cholesterol & Starch & Span 20 & $1: 15$ \\
\hline F24 & SPC & Cholesterol & Starch & Span 20 & $1: 25$ \\
\hline F25 & SPC & Cholesterol & Starch & Tween 80 & 1:05 \\
\hline F26 & SPC & Cholesterol & Starch & Tween 80 & $1: 15$ \\
\hline F27 & SPC & Cholesterol & Starch & Tween 80 & $1: 25$ \\
\hline
\end{tabular}

the funnel adjusted at circa $2-4 \mathrm{~cm}$ distance from the table surface. The weight of the powder was recorded (10 g) and poured into a funnel, where a uniform cone was formed (USP, 2015; BP, 2016b). Any asymmetric cone formation was discarded and the measurements were repeated again. The height of the powder cone (from base to the tip of the cone formed) and diameter of the powder base was measured. This procedure was repeated to determine AOR values for all three coarse carbohydrate carriers as well as protransfersome formulations (i.e. F1-F27) with the help of following equation;

Angle of Repose $\left({ }^{\circ}\right)=\tan ^{-1}\left(\frac{\text { Powder height }(\mathrm{cm})}{\text { Powder base }(\mathrm{cm})}\right)$

For Carr's consolidation index, a graduated cylinder of $25 \mathrm{ml}$ with $1 \mathrm{ml}$ increments was used (USP, 2015; BP, 2016c). Coarse carbohydrate carrier or protransfersome powder formulations $(10 \mathrm{~g})$ were transferred to the cylinder, followed by 2-3 gentle taps on a hard surface to ensure a uniform powder level in the cylinder. The initial volume $\left(\mathrm{V}_{0}\right)$ of powder in the cylinder was noted and the cylinder was then adjusted or set in an automated controlled tapping tapped density tester (Agilent technologies, USA). The height (14 $\pm 2 \mathrm{~mm})$ and tapping rate $(100 \pm 15$ tapes $/ \mathrm{min}$ for $5 \mathrm{~min})$ of the cylinder were set. After complete tapping, the final volume $\left(\mathrm{V}_{\mathrm{f}}\right)$ of the powder in the cylinder was recorded and Carr's consolidation index was determined with the help of an equation using initial volume and final tapped volume of powder in the cylinder (Carr, 1965). This experiment was repeated for other coarse carbohydrate carriers and all protransfersome powder formulations (F1-F27).

Car's $\operatorname{Index}(\%)=\left(\frac{\mathrm{V} 2-\mathrm{V} 1}{\mathrm{~V} 2}\right) \times 100$

\subsection{Characterisation of particles size and zeta potential of transfersome} vesicles

Protransfersome powders were hydrated $(150 \mathrm{mg} / 5 \mathrm{ml})$ in deionised water to form transfersome suspensions, followed by an annealing period of $1 \mathrm{~h}$. Annealing of hydrated transfersome was achieved by leaving the transfersomes without disruption/shaking at a temperature above the phase transition of the lipid. Annealing may help to overcome the possible structural defects of the transfersome bilayers (Lawaczeck et al., 1976). Transfersome particle size also known as volume median diameter (VMD) and poly dispersity index (PDI also referred to as SPAN) were measured using laser diffraction (Malvern Mastersizer 200, Malvern Instruments, UK). This instrument was used to measure particle size in a micrometre range.

Zeta potential of transfersome particles was determined using a Zetasizer Nanoseries (Malvern Instruments Ltd., UK) employing Laser Doppler Velocimetry (LDV) via electrophoretic mobility in dispersion medium. Transfersomes in the nanometre range were measured using dynamic light scattering (DLS) (Zetasizer Nano; Malvern Instruments Ltd., UK).

\subsection{Drug entrapment in transfersomes}

Transfersomes were generated from dry protransfersome formulations $(150 \mathrm{mg} / 5 \mathrm{ml})$, followed by annealing. For total drug, $1 \mathrm{ml}$ of the formulation was diluted with methanol and the PTX present was quantified using HPLC (Agilent 1200 HPLC instrument, UK). For the unentrapped PTX, $0.5 \mathrm{ml}$ of the formulation was transferred to a Millipore filter (10 KDa) (Fischer Scientific, UK) and subjected to bench centrifugation (Spectrafuge 24D, Labnet International, USA) for $30 \mathrm{~min}$ at $15,100 \mathrm{~g}$. This process facilitated the separation of PTX-entrapped transfersomes (i.e. the vesicles do not pass through the filter due to their large size) from the unentrapped PTX (filtrate, only allowing free PTX to pass). The unentrapped component passing through the filter was diluted with methanol to determine the entrapment of PTX in transfersomes using following equation:

Entrapment efficiency $(\%)=\left(\frac{\text { Total drug }- \text { Unentrapped drug }}{\text { Total drug }}\right) \times 100$

PTX quantification was achieved via HPLC, using acetonitrile and water $(80: 20 \% \mathrm{v} / \mathrm{v})$ as a mobile phase with a flow rate of $1.2 \mathrm{ml} / \mathrm{min}$ 
and a detection wavelength of $230 \mathrm{~nm}$. Machine temperature was set at $25{ }^{\circ} \mathrm{C}$, with an injection volume of $10 \mu \mathrm{l}$. The HPLC column used was a ODS C-18, $5 \mu \mathrm{m}$ and $150 \mathrm{~mm} \times 4.6 \mathrm{~mm}$ (Agilent technology, USA). A calibration curve of PTX $(5-80 \mu \mathrm{g} / \mathrm{ml})$ was constructed for the quantification of unknown PTX concentration.

\subsection{Morphology studies of protransfersome via scanning electron microscopy and transfersome vesicles via transmission electron microscopy}

Scanning electron microscopy (SEM) was employed to investigate the surface morphology of the dry protransfersome powder formulations. Whereas, transmission electron microscopy (TEM) was used to examine the structure of the transfersomes. For examination using SEM, protransfersome powders was transferred to aluminium stubs, followed by an air spray to remove any excessive powder from the stub. The stub samples were then coated with a thin layer of gold via an EMITECH K550X Fine Coater (Quorum Technologies, UK) for 2 min under vacuum. Protransfersome powders were then examined via SEM (Quanta 200 , Netherlands) at $20 \mathrm{kV}$ and the images at various magnifications were captured. For TEM analysis, a drop of formulation was placed on a carbon coated copper grid (400 mesh) (TAAB Laboratories Equipment Ltd., UK), followed by the addition of one drop of phosphotungstic acid (i.e. $1 \% \mathrm{w} / \mathrm{v}$, as negative staining). Transfersomes structure was observed with a number of images captured via a Philips CM 120 Bio-Twin TEM (Philips Electron Optics BV, Netherlands).

\subsection{Nano sizing of transfersome vesicles formulations followed by spray drying or freeze drying}

Upon investigation of $\mathrm{F} 1-\mathrm{F} 27$ protransfersome powder formulations, a range of formulations were selected based on the aforementioned characteristics (AOR, compressibility index, VMD, SPAN, zeta potential and entrapment efficiency). Transfersome suspensions posthydration and annealing were analysed in terms of size via a Malvern Mastersizer. Transfersome suspensions (vesicles within the micrometre size range) were reduced into the nanometre size range with the help of probe sonication (Q125 Qsonica, USA) and referred to as Nano formulations. Probe sonication was employed for $10 \mathrm{~min}$ with a higher amplitude intensity (i.e. 80\%).The resultant Nano transfersome vesicles suspensions were converted into dry powders via spray drying (i.e. spray dried nano; SDN) and freeze drying (i.e. freeze dried nano; FDN) formulations.

For spray drying (SD), Nano transfersome vesicles suspensions were fed into the Büchi Mini Spray Dryer B-290 connected with high performance cyclone (Büchi Laboratories, Switzerland). Atomization of transfersome suspensions was conducted using a $0.7 \mathrm{~mm}$ nozzle. Outlet temperature was set at $100{ }^{\circ} \mathrm{C}$ (i.e. inlet temperature of $50 \pm 3{ }^{\circ} \mathrm{C}$ ) with a feed rate of 5-6 $\mathrm{ml} / \mathrm{min}$. Aspiration and atomizing air flow rate was adjusted to $100 \%$ and $400 \mathrm{~L} / \mathrm{h}$ respectively. Transfersome suspensions were agitated (with the help of magnetic stirrer) prior to feeding into the spray dryer apparatus in order to yield uniform dry particles. The formed spray dried powders were collected from the collecting vessel and stored in a seal airtight bottle $(100 \mathrm{ml})$. Dry powders were stored in airtight containers in a desiccator for further studies.

For freeze drying (FD), Nano transfersome suspensions were poured into a $100 \mathrm{ml}$ glass bottle and placed in a freezer (Indesit, UK) overnight in order to form a solid frozen mass in bottle. Following this process, the frozen sample was then stored in an electronic freeze dryer (Beijer Electronics, Spain) (overnight) in order to determine the dry mass of protransfersome powders. These powder formulations were stored in airtight bottles $(100 \mathrm{ml})$ in a desiccator for further studies. The same procedure of spray drying (SD) and FD was repeated for all selected formulations.

Production yield was calculated (formula) as the weight of the final product with respect to the total weight of starting materials (i.e. protransfersome powder weight prior hydration) used in formulation. Where $\mathrm{W}_{1}$ is the final weight of powder formulation collected from the collecting vessel of spray drying and $\mathrm{W}_{2}$ is the initial weight of starting material used for protransfersome powder formulation.

Production Yield $(\%)=\left(\frac{\mathrm{W} 1}{\mathrm{~W} 2}\right) \times 100$

\subsection{Manufacturing of protransfersome tablets}

A Stylcam 2000R compaction simulator tabletting machine (Medelpharm, France) was employed for the manufacture of selected protransfersome powder formulations into tablets, ranging in weight from 80 to $250 \mathrm{mg}$. A $7 \mathrm{~mm}$ flat punch and die set was used in order to compress protransfersome powders into protransfersome tablets with the filling height adjusted to $8 \mathrm{~mm}$. An average compression force of $10 \mathrm{kN}$ was used with a manufacturing speed of 10 tablets per min. A temperature of $17{ }^{\circ} \mathrm{C}$ and relative humidity of $40 \%$ was maintained throughout the manufacturing process.

\subsection{Quality control tests of protransfersome tablets}

Protransfersome tablets were characterized using standard quality control tests including; weight uniformity, crushing strength, thickness and disintegration tests. These tests were conducted in order to assess their pass or fail criteria on the basis of BP guidelines.

In the weight uniformity of protransfersome tablet test, 20 randomly selected tablets were weighed individually from each batch using a calibrated balance (PI-225DA Denver Instruments, Germany). The average weight and the standard deviation were recorded (BP, 2016d).

A hardness tester (Dr Schleuniger/ Pharmatron, USA) was used to determine the crushing strength of the 10 randomly selected tablets from a batch of protransfersome tablets. Before the test, the hardness tester was calibrated and the crushing strength of the tablets measured in newtons.

For thickness testing, again 10 tablets were randomly selected from a batch of tablets with the help of a digital vernier calliper (Copley Scientific, UK). Tablets thickness was measured in millimetres (mm). Where the tablet diameter is lower than $12.55 \mathrm{~mm}$ a tolerance acceptance level of $\pm 5 \%$ is inferred; whereas if the tablet thickness is greater than $12.55 \mathrm{~mm}$, the acceptance level is $\pm 3 \%$ (Lachman et al., 1986).

In disintegration testing, 6 randomly selected tablets were transferred to the basket-rack assembly of a disintegration tester (PTZ Pharma test instruments, Germany). A beaker $(1000 \mathrm{ml})$ was previously filled with circa $800-900 \mathrm{ml}$ of deionised water as the disintegration medium, and water bath was adjusted at $37{ }^{\circ} \mathrm{C}$. Tablets in the basketrack assembly were immersed in the medium and agitated until complete disintegration was achieved (BP, 2016a).

\subsection{In-vitro studies of protransfersome tablet formulations via nebulization}

Two nebulizers, Vibrating mesh and Ultrasonic were employed in aerosolization studies in the in-vitro artificial lung model also known as Two Stage Impinger (TSI) (Copley Scientific, UK). TSI is comprised of two stages; the upper stage (i.e. representing the upper part of the respiratory tract) and the lower stage (i.e. representing the lower part of respiratory tract). The cut-off aerodynamic diameter between the upper stage and lower stages is $6.4 \mu \mathrm{m}$. For the collection of aerosol in the TSI, $7 \mathrm{ml}$ deionised water was placed in the upper stage and $30 \mathrm{ml}$ in the lower stage respectively. After assembling the parts of TSI, the air flow rate was set at $60 \mathrm{~L} / \mathrm{min}$. Protransfersome tablets of each selected formulation were hydrated in nebulizer reservoir (i.e. $150 \mathrm{mg} / 5 \mathrm{ml} \mathrm{w} / \mathrm{v}$ ratio) and positioned in front of the mouthpiece of TSI. Nebulization performance was characterized for both nebulizers in terms of 
nebulization time, sputtering time, mass output (\%) and output rate $(\mathrm{mg} / \mathrm{min})$ for all selected protransfersome tablet formulations. Nebulizer mass output and output rate was measured with the help of equations.

\section{Mass output (\%) $=\left(\frac{\text { Weight of nebulised formulation }}{\text { Weight of formulation present in the nebuliser prior to nebulisation }}\right) \times 100$}

Aerosol output rate $(\mathrm{mg} / \mathrm{min})=\left(\frac{\text { Weight of nebulised formulation }}{\text { Complete nebulisation time }}\right)$

\subsection{Cell viability assay using transfersome generated from protransfersome tablet formulations}

Normal cells i.e. MRC-5 (normal lung fibroblast cells) and cancer immortalized human lung fibroblast cells MRC-5 SV2 were used in this study to provide a proof of concept (Zhu and Gooderham, 2002; Huschtscha and Holliday, 1983). These cells were plated into black, flat-bottomed 96-well plates at a density of $1 \times 10^{4}$ cells $/ \mathrm{ml}(90 \mu \mathrm{l}$ / well), where growth medium Dulbecco's Modified Eagle Medium (DMEM) was used containing Foetal Bovine Serum (FBS) of $10 \%$, Lglutamine $1 \%$ and antibiotic-antimycotic solution of $1 \%$. MRC-5 and MRC- 5 SV2 cells were then incubated in a humidified atmosphere of $5 \%$ of $\mathrm{CO}_{2}$ at $37^{\circ} \mathrm{C}$ for $24 \mathrm{~h}$. After $24 \mathrm{~h}, 96$-well plates containing MRC-5 and MRC-5 SV2 cells were tested with $70 \%, 30 \%$ and $10 \%$ of transfersome suspension generated from protransfersome tablets formulation i.e. F3, F6 or F9 (i.e. PTX-free and PTX-loaded formulations for comparison). After incubation of $24 \mathrm{~h}$, Alamar Blue (AB) (10\%) was added in each well, and again incubated for $3 \mathrm{~h}$ (in order to quantify cell viability). Post incubation, plates were allowed to cool to room temperature and a microplate reader (Epoch, BioTek Instruments Ltd., Swindon, UK) was used for reading plates (fluorescence excitation at $545 \mathrm{~nm}$, and emission at $600 \mathrm{~nm}$ ). The obtained values from formulation F3, F6 or F9-treated wells were compared with control wells (i.e. treated with deionised water). Control values were set at $100 \%$ and the values of formulation F3, F6 or F9-treated wells were normalized to the control values.

\subsection{Statistical analysis}

One-way analysis of variance (ANOVA) or student's t-test was conducted in order to perform statistical assessments of difference (using SPSS software) amongst more than two groups or two sets of data respectively. All values were expressed as a mean \pm SD (standard deviation) and a $p$-value $<0.05$ was considered as a significant difference. All experiments were performed in quadruplicate.

\section{Results and discussion}

\subsection{Morphology of carbohydrate carrier using SEM}

SEM was used to examine surface morphology of coarse carbohydrate carriers i.e. LMH, MCC and Starch. Upon examination of LMH, the particles were found to be smaller and uniform in size and shape (Fig. 1a). LMH is typically employed as a filler/diluent in tablet manufacturing, thus these carrier particles offer enhanced flowability rolling with ease over each other without offering resistance to the movement and improve die filling, thus reducing weight variation in tablet manufacturing (Prescott and Barnum, 2000; Aulton and Taylor, 2018c) (Fig. 1a). LMH particles are non-porous and due to their smaller and uniform size and shape, the lipid phase is uniformly distributed over these carrier particles during protransfersome formulation (Elhissi et al., 2011; Khan et al., 2018).

MCC particles appeared to be irregular and variable in size and shape (Fig. 1b). MCC particles were composed of both crystalline and amorphous areas, with cellulose fibres tending to aggregate, thus having the appearance of round to oblong in shape (Aulton and Taylor, 2018c) (Fig. 1b). The difference in morphology of these particles may restrict particle flowability, which would ultimately affect die filling during tabletting. However, these particles possess good disintegration properties which may help in disintegration and dissolution of tablets (Aulton and Taylor, 2018c).

Starch coarse particles were also observed to be irregular in shape and size (very small to very large particles) (Fig. 1c). However in terms of morphology, Starch particles shapes were not oblong and therefore they appeared to have superior flowability when compared to the oblong MCC particles.

\subsection{Flow properties of protransfersome powder via AOR and compressibility index}

Upon analysis of AOR, coarse carbohydrate carriers demonstrated angles of $10.24 \pm 1.22,18.65 \pm 1.55$ and $15.01 \pm 1.16^{\circ}$ for LMH, MCC and Starch correspondingly, exhibiting excellent flowability in accordance to Carr's index (Carr, 1965) (Table 2). The incorporation or exclusion of PTX in the protransfersome powder formulations did not affect the flowability characteristics and therefore values originating from formulations without PTX were not included. PTX-loaded LMH protransfersome powder formulations F1-F9, demonstrated excellent flowability (angle between 25 and 30 ${ }^{\circ}$ (Table 2) (Carr, 1965). However, comparatively lower AOR were noted for formulations F3, F6 and F9 (all these formulations manufactured in 1:25 w/w lipid phase to carrier ratios) when compared to F1, F2, F4, F5, F8 and F7 powder formulations (prepared in a 1:05 or 1:15 lipid phase to carrier ratios) (Table 2). This difference in AOR between protransfersomes is related to the presence of lighter or denser lipid phase coatings over carbohydrate carriers (Table 1). The denser lipid phase coating may cause the coated powder to agglomerate and therefore resist the free movement of particles stacking upon each other as opposed to free flowing (Table3).

A similar trend in AOR was demonstrated by the MCC-based protransfersome (i.e. F10-F18) and Starch-based protransfersome (i.e. F19-F27) powder formulations, respectively (Table 2). Overall, the powder formulations (F10-F27) exhibited excellent powder flowability, with flowability and AOR increasing as carbohydrate ratio increased. MCC-based protransfersome powder formulations demonstrated comparatively higher angle of reposes than starch-based formulations, which may be attributed to the variance in particle size of MCC eliciting greater resistance to powder flow.

Moreover, LMH-based protransfersome powders offered superior flowability due to the associated smaller and uniform particle size, which enhanced flow of LMH particles when compared to MCC or Starch particles (Section 3.1). Upon analysis of compressibility index, coarse carbohydrate carriers demonstrated good flowability for LMH, compared to fair flowability of Starch and passable flowability of MCC carriers (Table 2) (Carr, 1965). This may be attributed to the carrier morphology of LMH (uniform and smaller particles), when compared to MCC (i.e. oblong particles) and Starch (irregular in size and shape particles) (Section 3.1). The aforementioned trend was mirrored with respect to compressibility index for the protransfersome powder formulations (F1-F27). In LMH-based protransfersome powder formulations (F1-F9), compressibility index indiciated excellent flowability for F1, F4 and F7 formulations, when compared to good flowability of F2, F3, F5, F6, F8 and F9 (Table 2). Significantly $(p<0.05)$ lower values of compressibility index of protransfersome powder was noted for formulations with a 1:05 w/w lipid phase to carrier ratio (i.e. F1, F4 and F7), when compared to formulations with a 1:25 w/w lipid phase to carrier ratio (F3, F6 and F9) (Table 2). This may be attributed to a denser lipid coat over the LMH carriers in the 1:05 w/w ratio formulations. The presence of high lipid content may reduce the carrier movement hindering flowability (Table 1 ). Hence, particles in these 

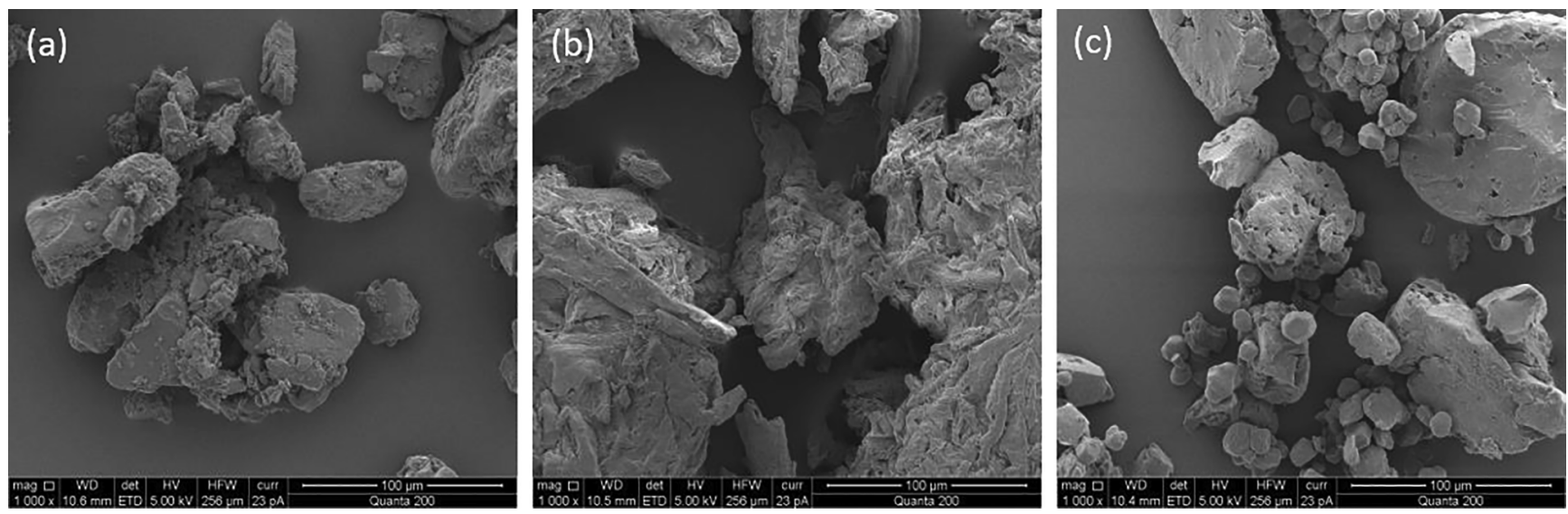

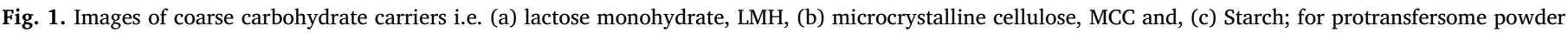
formulations. These images are typical of four different experiment.

formulations (F1, F4 and F7) may stick to each other during tapping, and occupy a lower volume, as particles are unable to sediment during tapping and maintain their volume in the graduated cylinder. Contrastingly, in protransfersome formulations which possessed an elevated proportion of carbohydrate carrier $(1: 15$ or $1: 25 \mathrm{w} / \mathrm{w})$, flowability was maintained due to the lower degree of particle resistance/ stickiness. This trend was also reflected in compressibility index values for MCC and starch-based protransfersome powder formulations (Table 2). However some differences were observed, with the MCC and starch-based protransfersome powder formulations i.e. 1:05 w/w demonstrated fair flowability when compared to passable flowability for 1:25 w/w formulations (Table 2). The observed fair and passable flowability may be associated with the particles morphology of MCC (i.e. oblong shaped particles with irregular in size) and starch (irregular size and shape) (Section 3.1).

Overall, it was identified that compressibility index for formulations with $1: 05 \mathrm{w} / \mathrm{w}$ demonstrated enhanced powder flowability. However, due to high lipid content compared to other formulation ratios (i.e. 1:15 or $1: 25 \mathrm{w} / \mathrm{w}$ ), the formulation particles elicited resistance to settling, maintaining the powder volume during tapping. These results are suggestive that a higher lipid content, would increase particle resistance and deceptively enhance flowability. However, in AOR studies, powder formulations with 1:25 w/w lipid phase to carrier ratio demonstrated better flowability with low angle and therefore would improve die

Table 2

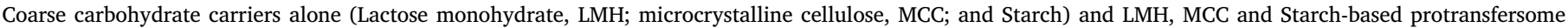

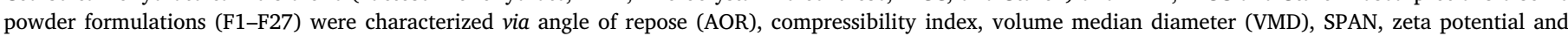
entrapment efficiency. Data are mean $\pm S D, n=4$.

\begin{tabular}{|c|c|c|c|c|c|c|}
\hline Formulation Number & *Angle of Repose $\left(^{\circ}\right)$ & *Compressibility Index (\%) & Volume Median Diameter $(\mu \mathrm{m})$ & SPAN & Zeta Potential (mV) & Entrapment Efficiency (\%) \\
\hline$L M H$ & $10.24 \pm 1.22$ & $13.85 \pm 3.95$ & $N / A$ & $N / A$ & $-3.68 \pm 0.74$ & $N / A$ \\
\hline$M C C$ & $18.65 \pm 1.55$ & $23.26 \pm 2.67$ & $N / A$ & $N / A$ & $-1.86 \pm 0.38$ & $N / A$ \\
\hline Starch & $15.01 \pm 1.16$ & $20.469 \pm 3.18$ & $N / A$ & $N / A$ & $-11.65 \pm 2.16$ & $N / A$ \\
\hline F1 & $18.45 \pm 1.16$ & $9.79 \pm 0.65$ & $5.34 \pm 0.77$ & $3.88 \pm 0.85$ & $-2.44 \pm 0.54$ & $97.25 \pm 3.18$ \\
\hline F2 & $15.06 \pm 1.22$ & $11.33 \pm 0.66$ & $6.53 \pm 0.67$ & $3.82 \pm 0.65$ & $-3.0 .7 \pm 1.21$ & $96.61 \pm 4.21$ \\
\hline F3 & $13.16 \pm 0.81$ & $12.21 \pm 0.54$ & $5.65 \pm 0.85$ & $2.51 \pm 0.62$ & $-2.39 \pm 0.44$ & $96.55 \pm 3.47$ \\
\hline F4 & $19.03 \pm 1.56$ & $9.88 \pm 0.65$ & $5.83 \pm 0.56$ & $4.06 \pm 0.85$ & $-3.17 \pm 1.13$ & $96.02 \pm 4.61$ \\
\hline F5 & $16.75 \pm 1.21$ & $11.24 \pm 0.52$ & $6.06 \pm 0.49$ & $3.76 \pm 0.68$ & $-3.46 \pm 0.85$ & $97.53 \pm 3.88$ \\
\hline F6 & $14.01 \pm 1.03$ & $12.06+0.31$ & $6.76 \pm 0.61$ & $3.75 \pm 0.55$ & $-3.09 \pm 0.60$ & $96.26 \pm 3.17$ \\
\hline F7 & $18.75 \pm 1.46$ & $9.77 \pm 0.38$ & $5.66 \pm 0.72$ & $3.65 \pm 0.77$ & $-3.02 \pm 0.92$ & $94.87 \pm 4.56$ \\
\hline F8 & $15.24 \pm 1.55$ & $11.45 \pm 0.44$ & $6.51 \pm 0.62$ & $3.44 \pm 0.67$ & $-2.41 \pm 0.46$ & $92.68 \pm 3.68$ \\
\hline F9 & $13.62 \pm 1.14$ & $12.50 \pm 0.73$ & $6.46 \pm 0.73$ & $4.11 \pm 0.96$ & $-3.04 \pm 0.66$ & $93.03 \pm 4.37$ \\
\hline F10 & $26.45 \pm 1.42$ & $19.75 \pm 1.20$ & $9.56 \pm 1.29$ & $2.85 \pm 0.89$ & $-3.28 \pm 0.78$ & $94.11 \pm 4.15$ \\
\hline F11 & $23.48 \pm 1.67$ & $20.46 \pm 0.85$ & $10.56 \pm 0.72$ & $3.76 \pm 0.77$ & $-4.03 \pm 0.84$ & $96.94 \pm 4.01$ \\
\hline F12 & $20.21 \pm 1.12$ & $22.79 \pm 1.16$ & $9.88 \pm 0.91$ & $3.63 \pm 0.79$ & $-3.84 \pm 1.47$ & $95.60 \pm 3.05$ \\
\hline F13 & $27.09 \pm 1.85$ & $18.87 \pm 0.83$ & $9.55 \pm 1.06$ & $3.76 \pm 0.88$ & $-2.52 \pm 0.55$ & $97.81 \pm 3.17$ \\
\hline F14 & $23.88 \pm 1.65$ & $20.68 \pm 1.78$ & $10.65 \pm 0.88$ & $2.99 \pm 0.67$ & $-2.18 \pm 1.04$ & $95.66 \pm 3.89$ \\
\hline F15 & $21.22 \pm 1.10$ & $21.26 \pm 1.84$ & $10.72 \pm 0.76$ & $3.81 \pm 0.77$ & $-3.42 \pm 0.95$ & $96.41 \pm 4.24$ \\
\hline F16 & $26.97 \pm 1.55$ & $19.34 \pm 1.53$ & $9.82 \pm 1.61$ & $3.45 \pm 0.68$ & $-3.14 \pm 0.76$ & $92.04 \pm 4.56$ \\
\hline F17 & $22.84 \pm 1.68$ & $22.16 \pm 1.66$ & $9.80 \pm 1.46$ & $3.80 \pm 0.55$ & $-2.98 \pm 1.25$ & $92.86 \pm 4.97$ \\
\hline F18 & $20.57 \pm 1.23$ & $22.89 \pm 1.73$ & $10.26 \pm 0.95$ & $3.56 \pm 0.75$ & $-3.42 \pm 0.73$ & $93.03 \pm 3.93$ \\
\hline F19 & $24.26 \pm 1.22$ & $16.25 \pm 1.42$ & $11.02 \pm 1.06$ & $4.46 \pm 1.26$ & $-3.70 \pm 1.35$ & $94.90 \pm 4.01$ \\
\hline F20 & $20.27 \pm 1.31$ & $18.46 \pm 1.61$ & $12.56 \pm 1.41$ & $4.36 \pm 1.33$ & $-4.10 \pm 1.20$ & $97.16 \pm 3.17$ \\
\hline F21 & $18.53 \pm 0.82$ & $21.34 \pm 1.76$ & $9.56 \pm 0.95$ & $3.22 \pm 0.74$ & $-3.58 \pm 1.05$ & $96.62 \pm 4.57$ \\
\hline F22 & $23.73 \pm 1.17$ & $16.91 \pm 1.72$ & $10.68 \pm 0.86$ & $4.45 \pm 1.49$ & $-3.91 \pm 0.94$ & $95.70 \pm 3.16$ \\
\hline F23 & $20.44 \pm 1.26$ & $19.65 \pm 1.85$ & $9.62 \pm 1.05$ & $3.46 \pm 0.76$ & $-4.23 \pm 1.67$ & $97.05 \pm 3.97$ \\
\hline F24 & $19.30 \pm 1.03$ & $21.19 \pm 1.79$ & $10.16 \pm 0.79$ & $3.73 \pm 0.82$ & $-3.09 \pm 0.83$ & $96.78 \pm 4.54$ \\
\hline F25 & $25.68 \pm 1.65$ & $16.91 \pm 1.75$ & $11.39 \pm 0.88$ & $3.96 \pm 077$ & $-3.23 \pm 0.97$ & $94.92 \pm 3.77$ \\
\hline F26 & $24.13 \pm 1.95$ & $18.68 \pm 1.69$ & $9.82 \pm 1.12$ & $2.33 \pm 0.86$ & $-3.52 \pm 0.89$ & $94.96 \pm 4.62$ \\
\hline F27 & $22.12 \pm 1.11$ & $22.39 \pm 1.86$ & $10.76 \pm 1.31$ & $4.23 \pm 1.23$ & $-4.76 \pm 1.33$ & $93.81 \pm 3.30$ \\
\hline
\end{tabular}

* Flow properties of powder employing angle of repose (AOR) and compressibility index (CI) via Carr's scale of flowability (Carr, 1965) show the following flow character; excellent (25-30 AOR, 1-10 CI), good (31-35 AOR, 11-15 CI), fair (36-40 AOR, 16-20 CI), passable (41-45 AOR, 21-25 CI), poor (46-55 AOR, 26-31 CI), very poor (56-65 AOR, 32-37 CI) and very very poor ( $>65 \mathrm{AOR},>38 \mathrm{CI}$ ). 
Table 3

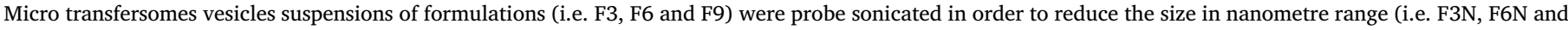

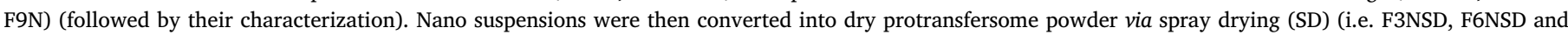

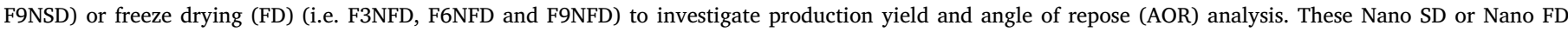

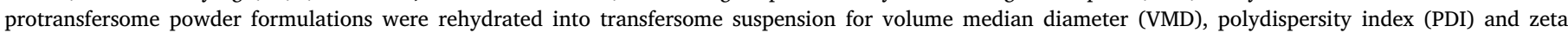
potential analysis. Data are mean $\pm \mathrm{SD}, \mathrm{n}=4$.

\begin{tabular}{|c|c|c|c|c|c|}
\hline Formulations & VMD (nm) & PDI & Zeta potential (mV) & Production yield (\%) & $\operatorname{AOR}\left({ }^{\circ}\right)$ \\
\hline \multicolumn{6}{|c|}{ After Probe Sonication } \\
\hline F3N & $254.36 \pm 5.64$ & $0.382 \pm 0.67$ & $-2.55 \pm 0.65$ & N/A & N/A \\
\hline F6N & $458.92 \pm 7.78$ & $0.330 \pm 0.73$ & $-2.49 \pm 0.74$ & N/A & N/A \\
\hline F9N & $321.56 \pm 7.44$ & $0.368 \pm 0.66$ & $-2.63 \pm 0.77$ & N/A & N/A \\
\hline \multicolumn{6}{|c|}{ After Spray Drying } \\
\hline F3NSD & $261.51 \pm 6.85$ & $0.421 \pm 0.54$ & $-2.67 \pm 0.62$ & $57.94 \pm 2.86$ & $46.65 \pm 2.06$ \\
\hline F6NSD & $465.85 \pm 6.89$ & $0.397 \pm 0.61$ & $-2.53 \pm 0.75$ & $60.99 \pm 3.88$ & $49.68 \pm 1.62$ \\
\hline F9NSD & $316.92 \pm 8.25$ & $0.413 \pm 0.59$ & $-2.62 \pm 0.69$ & $63.86 \pm 3.69$ & $48.54 \pm 1.43$ \\
\hline \multicolumn{6}{|c|}{ After Freeze Drying } \\
\hline F3NFD & $292.16 \pm 5.43$ & $0.451+0.71$ & $-2.55 \pm 0.76$ & $93.52 \pm 3.12$ & - \\
\hline F6NFD & $483.62 \pm 5.67$ & $0.434+0.69$ & $-2.61 \pm 0.68$ & $93.48 \pm 3.62$ & - \\
\hline F9NFD & $362.44 \pm 7.16$ & $0.444+0.58$ & $-2.58 \pm 0.76$ & $94.76 \pm 3.55$ & - \\
\hline
\end{tabular}

filling during tablet manufacturing. Therefore, protransfersome powder formulations based on LMH were recognised as superior, when compared to MCC and starch-based formulations. On the basis of the abovementioned results, LMH-based protransfersome powder formulations (i.e. F3, F6 and F9) were chosen for further studies.

\subsection{VMD, SPAN, zeta potential and entrapment efficiency of PTX in transfersome suspension}

Transfersomes were generated from PTX-loaded protransfersomes in deionised water via hydration followed by annealing (Section 2.4). Size, also referred to as volume median diameter (VMD) of transfersomes was analysed for all formulations (F1-F27). Size of transfersomes generated from protransfersomes were within the micrometre range, thus these formulations (i.e. F1-F27) were referred to as Micro protransfersomes formulations (Table 2). Upon analysis of VMD, LMHgenerated transfersomes (F1-F9) were significantly smaller $(p<0.05)$ when compared to transfersomes generated from MCC and Starchbased protransfersomes (F10-F27) (Table 2). Smaller VMD may be associated with their smaller particle size (resulting in a uniform distribution of lipid phase over carrier particles) and hypertonic environment caused by LMH, which cause shrinkage of lipid vesicle (Lehtonen and Kinnunen, 1994).

SPAN values generated from transfersome suspensions (i.e. F1-F27) exhibited no significant difference $(p>0.05)$, with values ranging from $2.33 \pm 0.86-4.45 \pm 0.99$ (Table 2). Transfersome SPAN values were not influenced by carrier selection (LMH, MCC and Starch) or lipid phase to carrier ratios (i.e. 1:05, 1:15 and 1:25 w/w). A similar trend was exhibited in terms of zeta potential values; with no significant difference $(p>0.05)$ observed when transfersomes were generated from LMH, MCC or Starch-based protransfersome powder formulations (Table 2).

PTX entrapment efficiency was analysed via HPLC for all (F1-F27) formulations. The entrapment efficiency of PTX found was within the range of $92.04 \pm 4.56-97.81 \pm 3.17 \%$ (i.e. F1-F27) and no significant difference $(p>0.05)$ was observed (Table 2). These results are analogous to previous research findings, where the entrapment efficiency was as high as $91.65 \%$ in lipid-based formulations (You et al., 2017). Similarly, high entrapment efficiency circa $89 \%$ was also reported by Rane and Prabhakar (2009). Paolino et al. (2012) demonstrated $57 \%$ of entrapment efficiency using Phosphatidylcholine as a phospholipid, in ultra-deformable lipid-based vesicles. High entrapment efficiency of lipophilic PTX may be associated with the long hydrocarbon chains of SPC, where PTX molecules may be easily accommodated (Table 2). Additionally, phospholipid hydrophobic chains may also enhance the solubilisation of PTX in the concentric bilayers, enhancing drug entrapment (i.e. like dissolves like). SPC is a natural phospholipid and it is both biocompatible and biodegradable and its toxicity is extremely low (Di Sotto et al., 2018; Nguyen et al., 2017). Furthermore, it offers lower phase transition temperature (i.e. -20 to $-30^{\circ} \mathrm{C}$ ), which makes this phospholipid more suitable to self-assemble into vesicles at room temperature or lower (Taylor and Morris, 1995). Surfactants are one of the basic components of transfersome/protransfersome formulations, making vesicles elastic/flexible, thus these vesicles are referred to as flexible or elastic vesicles. Three different surfactants based on their various HLB values were used in order to study their effect on size, zeta potential and ability to entrap paclitaxel. However, surfactant type did not significantly change the performance of vesicles in terms of size, charge and entrapment efficiency as compared to carrier type.

\subsection{Initial selection of Micro protransfersome formulations for further testing and tablet manufacturing}

Initial investigation and characterization was conducted for all PTXloaded Micro protransfersome powder formulations (i.e. F1-F27) including; AOR and compressibility index. VMD, SPAN, zeta potential and entrapment efficiency of PTX were determined for transfersomes hydrated from protransfersome powder formulations (Sections 3.2 and 3.3). Upon analysis of flow properties of protransfersome powder formulations, AOR and compressibility index values were indicative that LMH-based protransfersome formulations were superior.

\subsection{Examination of transfersome vesicles via TEM}

Selected LMH-based protransfersome powder formulations i.e. F3, F6 and F9 were hydrated and their morphology observed. Images captured suggested that transfersomes were spherical in shape and appeared to be multilamellar, but the artefacts of TEM including dehydration of sample may have resulted in rupture of large multilamellar vesicles whilst the smaller vesicles (oligolamellar vesicles and unilamellar vesicles) survived the stressful environment of TEM. (Fig. 2). These images were confirmative that transfersomes were successfully formulated.

\subsection{Characterization of spray drying and freeze drying after size reduction}

Upon initial analysis, three protransfersome formulations (F3, F6 and F9) were hydrated to generate Micro transfersome vesicles. These micrometre vesicles were then reduced to nanometre $(\mathrm{N})$ size (i.e. F3N, 

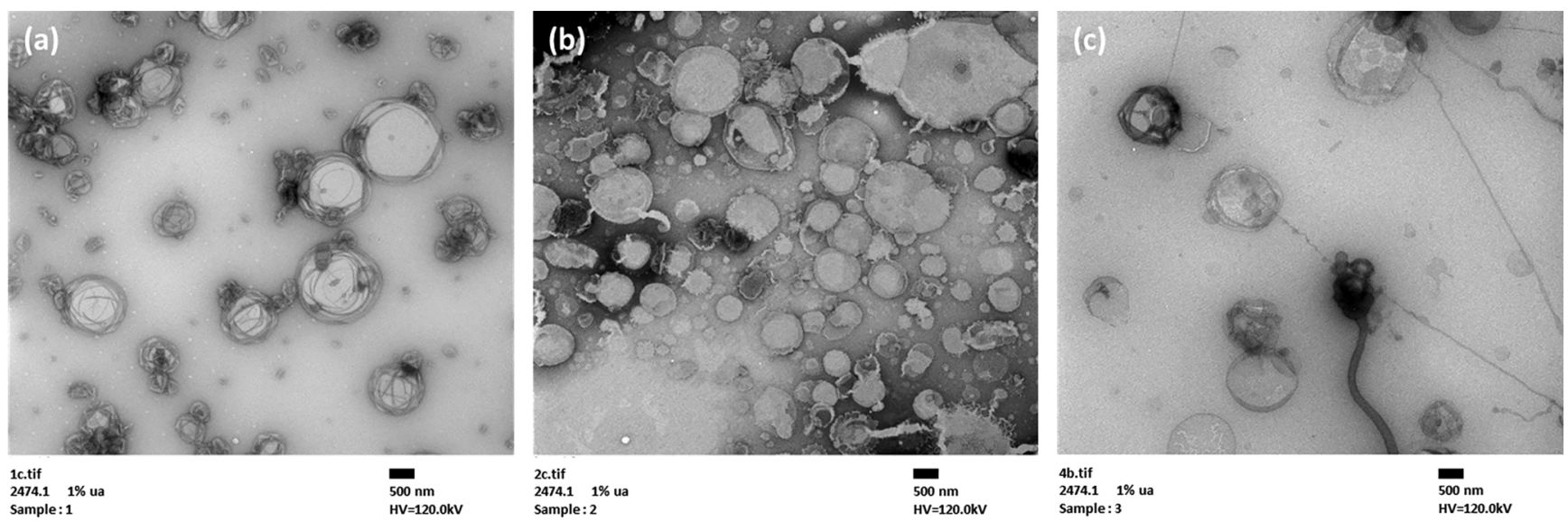

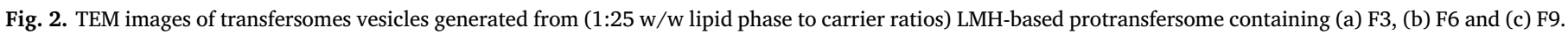
These images are typical of four different experiments.

F6N and F9N) via probe sonication, followed by their conversion into dry protransfersome powder formulations via spray drying (SD) (F3NSD, F6NSD and F9NSD) or freeze drying (FD) (F3NFD, F6NFD and F9NFD). In summary nine formulations were taken forward for further studies.

The initial VMD of Micro size transfersomes i.e. F3, F6 and F9 (5.65 $\pm 0.85,6.76 \pm 0.61$ and $6.46 \pm 0.73 \mu \mathrm{m}$ ) (Table 2) was significantly $(p<0.05)$ reduced to Nano size $(254.36 \pm 5.64$, $458.92 \pm 7.78$ and $321.56 \pm 7.44 \mathrm{~nm}$ ) via probe sonication (Table 3). Following SD, Nano SD protransfersome powder formulations (F3NSD, F6NSD and F9NSD) were rehydrated into transfersomes, and no significant difference ( $p>0.05$ ) was determined in VMD before and after spray drying (Table 3). Contrastingly, FD rehydrated protransfersome formulations (F3NFD, F6NFD and F9NFD) exhibited significantly $(p<0.05)$ larger VMD when compared to the VMD of transfersome suspensions prior to freeze drying (following probe sonication) (Table 3). This increase in VMD of FD formulations (Yang et al., 2013; Hua et al., 2003) may be attributed to the formation of crystalline structure of water molecules during the freezing phase and therefore may disrupt the vesicle bilayer membrane. Thus, following rehydration, vesicles may aggregate or fuse together. Upon investigation of PDI and zeta potential values, no significant difference $(p>0.05)$ was observed between probe-sonicated transfersomes and transfersomes rehydrated following SD and FD (Table 3).

Production yield of Nano SD transfersome powder was calculated from the formulations collected in the collecting vessel of spray drying instrument. Low production yield (i.e. 57.94 \pm 2.86-63.86 \pm 3.69 ) and no significant difference ( $p>0.05$ ) was observed between F3NSD, F6NSD and F9NSD formulations (Table 3). This lower production yield may be attributed to the lower inlet temperature (i.e. $100{ }^{\circ} \mathrm{C}$ ). Low inlet temperatures may cause particles to deposit in the cyclone compartment due to poor droplet drying in the drying chamber increasing particle adherence to the walls of drying chamber and cyclone compartment (Maa et al., 1997, Maury et al., 2005; Imtiaz-Ul-Islam Langrish, 2009). Powder recovery post spray drying process may also depend upon the free flowing properties of powder. High moisture content at low outlet temperature may further causes sticking of powder particles to the chamber walls and therefore lower powder output. Contrastingly, a high air inlet temperature may increase the production yield (Rathananand et al., 2007), which may be attributed to heat energy available in the drying chamber responsible for quick drying of atomized droplets preventing sticking of particles to the walls of the spray dryer. AOR studies conducted for Nano SD powder formulations (F3NSD, F6NSD and F9NSD) exhibited poor flowability $\left(46.65 \pm 2.06,49.68 \pm 1.62\right.$ and $48.54 \pm 1.43^{\circ}$ ) (Table 3). This may be associated with amorphous and crystalline regions of the SD powder and the presence of moisture, which may further facilitate particle aggregation.

Alternatively, the obtained Nano FD powder formulations (i.e. F3NFD, F6NFD and F9NFD) exhibited a high production yield of $93.52 \pm 3.12,93.48 \pm 3.62$ and $94.76 \pm 3.55 \%$, however they were observed to be cohesive in form as a solid mass. Regardless of high production yield, the absence of flowability of the powder renders the formulation unsuitable for tabletting and was hence eliminated from further studies.

In summary, initial Micro formulations F3, F6 and F9 as well as Nano SD formulations F3NSD, F6NSD and F9NSD were selected for further investigations, demonstrating excellent $(13.16+0.81$, $14.01+1.03$ and $13.62+1.14^{\circ}$ ) (Table 2) to poor (i.e. $46.65 \pm 2.06$, $49.68 \pm 1.62$ and $48.54 \pm 1.43^{\circ}$ ) flow properties in terms of AOR (Table 3).

\subsection{Weight uniformity test of PTX-loaded protransfersome tablet}

PTX-loaded Micro protransfersome tablet formulations F3, F6 and F9 exhibited no significant difference $(p>0.05)$ in weight (Table 4). Similarly, no weight variation was recorded between PTX-loaded Nano SD protransfersome powders into tablets (Table 4). According to British pharmacopeia (BP), to comply with weight variation test, no more than two individual tablets should deviate from the average weight by more

Table 4

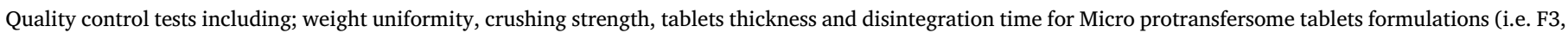
F6 and F9) as well as for Nano spray dried (SD) protransfersome tablets formulations (i.e. F3NSD, F6NSD and F9NSD). Data are mean \pm SD, $n=4$.

\begin{tabular}{|c|c|c|c|c|}
\hline Formulations & Weight uniformity (mg) & Crushing strength $(\mathrm{N})$ & Thickness (mm) & Disintegration Time (min) \\
\hline F3 & $138.64 \pm 6.85$ & $17.54 \pm 2.15$ & $2.62 \pm 0.41$ & $13.54 \pm 1.64$ \\
\hline F6 & $141.76 \pm 5.28$ & $18.36 \pm 2.82$ & $2.58 \pm 0.34$ & $12.43 \pm 1.71$ \\
\hline F9 & $135.89 \pm 6.74$ & $17.52 \pm 2.16$ & $2.67 \pm 0.39$ & $14.57 \pm 1.62$ \\
\hline F3NSD & $111.46 \pm 5.46$ & $10.52 \pm 1.26$ & $1.72 \pm 0.28$ & $7.64 \pm 1.06$ \\
\hline F6NSD & $103.32 \pm 6.71$ & $11.42 \pm 1.56$ & $1.83 \pm 0.24$ & $8.66 \pm 1.54$ \\
\hline F9NSD & $107.43 \pm 5.16$ & $12.64 \pm 1.34$ & $1.67 \pm 0.23$ & $8.74 \pm 1.63$ \\
\hline
\end{tabular}


than the percentage allowed i.e. $\pm 7.5 \%$ (i.e. when tablet weight is between 80 and $250 \mathrm{mg}$ ), and none of the tablets should deviate by more than twice this percentage (i.e. $\pm 15 \%$ ) (BP, 2016d). The average weight difference between Micro protransfersome tablets (F3, F6 and F9) and Nano SD tablets (F3NSD, F6NSD and F9NSD) formulations are significantly different $(p<0.05)$ from each other. This lower weight of Nano SD tablet formulation may be attributed to the combination of amorphous and crystalline regions of SD powder (i.e. a hygroscopic powder which also possesses moisture due to low outlet temperature during spray drying) which may facilitate powder aggregation and therefore impact upon die filling, resulting in lower tablet weight. Moreover, SD powder formulations also showed capping due to the sticky nature of SD powders during tabletting, lengthening tabletting process.

With respect to tablet weight uniformity, formulations F3 and F6 exhibited a deviation of two tablets, whereas F9 formulations demonstrated a deviation of 1 tablet from the average weight by $\pm 7.5 \%$; these formulations matched the acceptance criteria in accordance to BP standards (BP, 2016d). Comparatively, Nano SD formulations exhibited a deviation of 5 tablets for each F3NSD and F6NSD formulation, and 6 tablets for F9NSD from the average weight by $\pm 7.5 \%$ (not more than two tablets should deviate from the average weight) (Table 4). Thus, these three batches of SD tablets (i.e. F3NSD, F6NSD and F9NSD) failed the weight variation test in accordance to BP standards. These results were also substantiated by AOR values for the powder formulations; F3, F6 and F9 demonstrated excellent flow properties, whilst poor flow properties were observed for SD formulations (i.e. F3NSD, F6NSD and F9NSD) (Tables 2 and 3).

\subsection{Crushing strength of PTX-loaded protransfersome tablet}

Manufactured PTX-loaded Micro protransfersome tablets formulations F3, F6 and F9 demonstrated high crushing strength, whilst PTXloaded Nano SD tablets formulations (F3NSD, F6NSD and F9NSD) exhibited reduced hardness. However, hardness of SD tablets was significantly lower $(p<0.05)$ when compared to Micro protransfersome tablets formulations (Table 4). High hardness values of F3, F6 and F9 tablets formulations were related to the excellent flow properties (demonstrated by AOR) of the powders, which in turn enhance die filling compared to the poor flow properties of SD formulations (F3NSD, F6NSD and F9NSD) (Tables 2 and 3). Poor powder flowability may diminish die filling and consequently a lower compression force is used (due to low powder volume in die cavity) to compress powder into tablet. The high crushing strength of F3, F6 and F9 formulations, are indicative that these tablets may be able to withstand transportation and storage.

\subsection{Thickness and disintegration of PTX-loaded protransfersome tablet}

Die filling was previously adjusted to $8 \mathrm{~mm}$ to accommodate uniform powder filling. PTX-loaded Micro protransfersome tablets demonstrated a thickness measurements of $2.62 \pm 0.41,2.58 \pm 0.34$ and $2.67 \pm 0.39 \mathrm{~mm}$ for formulations F3, F6 and F9; with no significant difference upon comparison of the three formulations $(p>0.05)$ (Table 4). Similarly, no significant difference $(p>0.05)$ was also observed in tablet thickness amongst Nano SD tablet formulations. Interestingly, tablet thickness of protransfersome Micro formulations were significantly $(p<0.05)$ higher than Nano SD formulations; this is supported by the excellent and poor flowability of the corresponding powder particles (Tables 2 and 3). Moreover, weight variation tests also demonstrated that enhanced powder flow may facilitate complete die filling, as compared to inconsistent die filling demonstrated by agglomerated and hygroscopic SD powder formulations.

Upon investigation of disintegration time, PTX-loaded Micro protransfersome tablets exhibited longer disintegration time, which is directly associated with high compression force used to compress powders, also substantiated by the high tablet thickness and high crushing strength (Table 4). Contrastingly, low disintegration time was demonstrated by Nano SD tablet formulations (Table 4). Upon comparison, significantly higher $(p<0.05)$ disintegration times were found for Micro protransfersome tablet formulations when compared to Nano SD protransfersome tablets formulations. This lower disintegration time may be attributed to the nature of SD powder, which was hygroscopic facilitating water absorption succeeded by quick disintegration. Additionally, the lower weight and thickness of SD tablets may also improve tablet disintegration time. According to the BP standards, normal tablets should disintegrate within $15 \mathrm{~min}$, with no residue remaining in the disintegration instrument (BP, 2016a). Consequently, all protransfersome tablet formulations (i.e. F3, F6, F9, F3NSD, F6NSD and F9NSD) passed the disintegration test. Novel protransfersome tablets were characterized using various quality control tests (i.e. uniformity of weight, crushing test, thickness and disintegration time) in order to identify quality of the tablets. The sustained release of paclitaxel and other hydrophobic drugs from vesicles having similar composition is established in literature (Najlah et al., 2018; Han et al., 2018). These studies demonstrated evidence of release of paclitaxel in a controlled manner. Thus, we proposed that the key limiting step in achieveing sustained release is the disintegration of tablet, as a consequence this research primarily focused on tablet quality control tests.

Overall, after conducting quality control tests in accordance to BP; tablets manufactured from Micro protransfersome formulations F3, F6 and F9 passed all tests, demonstrating uniform weight variation and tablets thickness, high crushing strength and acceptable disintegration time). Whereas, Nano SD tablet formulations (i.e. F3NSD, F6NSD and F9NSD) failed the weight uniformity test and demonstrated low crushing strength; thus being eliminated from further studies. Based on these quality control tests, Micro protransfersome tablet formulations F3, F6 and F9 were further investigated for nebulization studies.

\subsection{Nebulization performance of PTX-loaded protransfersome tablets via Ultrasonic and Vibrating mesh nebulizers}

Ultrasonic and Vibrating mesh nebulizers were employed for the nebulization time of transfersome suspensions $(5 \mathrm{ml})$ generated from PTX-loaded Micro protransfersome tablet formulations (i.e. F3, F6 and F9). Upon analysis, using individual nebulizers (Ultrasonic or Vibrating mesh), no significant difference ( $p>0.05$ ) was noted between F3, F6 and F9 formulations (Table 5). However, nebulization time for the U1trasonic nebulizer was significantly shorter $(p<0.05)$ when compared to the Vibrating mesh nebulizer (Table 5). The lipid phase (i.e. SPC and cholesterol in 1:1 molar ratio) and carbohydrate ratio (3250 mg; 1:25 w/w lipid phase to carrier ratio) in formulations F3, F6 and F9 may enhance suspension viscosity (Elhissi et al., 2006; Elhissi et al., 2007); thus influencing the low energy of atomization used by Omron nebulizers, resulting in a longer nebulization time (Ghazanfari et al., 2007). Additionally, the large size of transfersome vesicles may also block the aperture of mesh (i.e. Omron nebulizer) and hence lengthen the nebulization time. Ultrasonic nebulizers function on the basis of piezoelectric crystal movement, which may be responsible for the formation of aerosols without impacting upon transfersome vesicle size and achieving a subsequently short nebulization time. Analogous results of shorter nebulization times achieved via Ultrasonic nebulizer was also reported by Mercer (1981) and Mc Callion et al. (1996), when compared to Omron nebulizer.

Similar results were also noted for sputtering time, where a significant difference $(p<0.05)$ was observed between Ultrasonic and Vibrating mesh nebulizers (Table 5). It was identified that viscosity of transfersome formulation suspension had a significant effect $(p<0.05)$ on the sputtering time, this may be attributed to the slow and consistent propagation of formulation through the mesh from the nebulizer reservoir in the Vibrating mesh nebulizer (over a long time 
Table 5

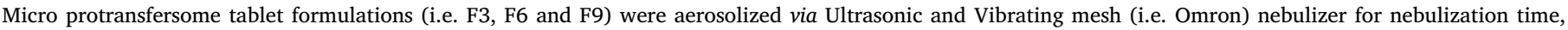
sputtering time, mass output and output rate. Data are mean $\pm S D, n=4$.

\begin{tabular}{|c|c|c|c|c|}
\hline Formulations \& Nebulization type & Nebulization time (min) & Sputtering time (min) & Mass output (\%) & Output rate $(\mathrm{mg} / \mathrm{min})$ \\
\hline \multicolumn{5}{|l|}{ Ultrasonic } \\
\hline F3 & $8.53 \pm 2.81$ & $2.42 \pm 0.32$ & $92.77 \pm 3.72$ & $460.33 \pm 10.65$ \\
\hline F6 & $9.15 \pm 2.66$ & $2.37 \pm 0.21$ & $90.65 \pm 4.51$ & $445.86 \pm 12.52$ \\
\hline F9 & $8.95 \pm 2.44$ & $2.62 \pm 0.28$ & $93.26 \pm 4.18$ & $464.42 \pm 10.72$ \\
\hline \multicolumn{5}{|l|}{ Vibrating mesh } \\
\hline F3 & $21.56 \pm 2.51$ & $1.62 \pm 0.23$ & $96.66 \pm 3.75$ & $200.67 \pm 9.84$ \\
\hline F6 & $23.12 \pm 2.66$ & $1.38 \pm 0.29$ & $97.18 \pm 2.62$ & $190.42 \pm 8.50$ \\
\hline F9 & $24.52 \pm 2.74$ & $1.26 \pm 0.29$ & $97.68 \pm 2.68$ & $181.06 \pm 10.46$ \\
\hline
\end{tabular}

period). Whereas in Ultrasonic nebulizer, transfersome suspensions stayed in the nebulizer (also referred to as residual or dead volume, which may not able to nebulize) may unable to form capillary waves followed by fragmentation (mechanism of Ultrasonic nebulizer for aerosolization). As a consequence, sputtering time was lengthened to aerosolise the residual volume. Similar results of sputtering time were also reported by Steckel and Eskandar (2003).

In mass output, regardless of complete nebulization time, $100 \%$ mass output cannot be achieved as a proportion of the formulation remains in the nebulizer reservoir (Clay et al., 1983). A lower mass output was demonstrated by Ultrasonic nebulizer when compared to Vibrating mesh nebulizer (Table 5). The lower mass output percentage of F3, F6 and F9 by Ultrasonic nebulizer may be attributed to the comparatively high proportion of dead volume (Table 5). Contrastingly, higher mass output by Vibrating mesh nebulizer for formulation suspension (F3, F6 and F9) may be attributed to the lower proportion of dead volume and longer nebulization time. Moreover, a lower dead volume was also reported by Vecellio et al. (2011) as well as Dolovich and Dhand (2011) when compared to Ultrasonic nebulizer. The higher mass output via Vibrating mesh nebulizer is in agreement with the previous research conducted by Elhissi and Taylor (2005) and Elhissi et al. (2006). Upon investigation of output rate, a significant difference $(p<0.05)$ was determined when employing Ultrasonic and Vibrating mesh nebulizers. The Ultrasonic nebulizer exhibited an output rate of more than double that of the Vibrating mesh nebulizer for formulations F3, F6 and F9 (Table 5). The lower output rate exhibited by the Vibrating mesh nebulizer may be attributed to the viscosity of transfersome suspension (blocking mesh aperture and increasing nebulization time). Contrastingly, the Ultrasonic nebulizer was least affected by the viscosity of transfersome suspension resulting in high output rate of formulations.

Overall, on the basis of nebulizer performance, the Ultrasonic nebulizer elicited shorter nebulization times and an average of $90 \%$ mass output. Whilst comparing to the Vibrating mesh nebulizer, the Ultrasonic nebulizer demonstrated approximately a two fold increase in output rate. Thus, the Ultrasonic nebulizer was identified as superior in performance when compared to Vibrating mesh nebulizer.

\subsection{Cell viability/cytotoxicity studies using transfersome generated from protransfersome tablet formulations}

In order to find a quick and simple cell viability study (i.e. proof of principle), MRC-5 (i.e. normal lung fibroblast cells) and cancer immortalized human lung fibroblast cells MRC-5 SV2 were employed for the cytotoxicity of PTX-free and PTX-loaded in protransfersome tablet formulations (i.e. F3, F6 and F9). MRC-5 SV2 cells are essentially a transformed version of MRC-5, where MRC- 5 cells were transfected with Simian virus SV-40 and thus immortalized (Huschtscha and Holliday, 1983). Non-transformed human lung fibroblasts have a limited lifespan when cultured in-vitro. Whereas, MRC-5 SV2 does not deteriorate and their growth does not slow down, indicating these cells have a potential for infinite growth (Yang et al., 1992). Moreover, the effect of carcinogenic agent was also studied using MRC-5 SV2 immortalized human lung fibroblast cells (as a potential lungs cancer cells) (Zhu and Gooderham, 2002). Both MRC-5 and MRC-5 SV2 cells were used for cytotoxicity studies using Alamar Blue assay. The effect of PTX-free and PTX-loaded F3, F6 and F9 tablet formulations on the viability of MRC-5 and MRC-5 SV2 cells were investigated over $24 \mathrm{~h}$ using three different formulation concentrations (i.e. $70 \%, 30 \%$ and $10 \%)$. PTX-loaded transfersome suspensions of $70 \%$ and $30 \%$ generated from protransfersome tablets were toxic to MRC- 5 SV2 cells but also significantly toxic to the normal MRC-5 cells too (these results were excluded and not shown), and therefore formulations with only $10 \%$ were plotted against time (24 h) and discussed (Fig. 3).

Employing PTX-free tablet formulations, the cell viability of both cells were unaffected $(\mathrm{p}>0.05)$, indicating that the preparation method and formulations parameters of protransfersomes (i.e. on MRC5 and MRC-5 SV2) had no cytotoxic effect. Similarly, with 10\% of PTXloaded transfersome suspension generated from protransfersome tablets (F3, F6 and F9) were safe in MRC-5 cells (Fig. 3). Alternatively, when using them (PTX-loaded F3, F6 and F9 formulations), they were noted to be significantly ( $p<0.05$ ) toxic to MRC- 5 SV2 cells, exhibiting cell viability of 60,68 and $67 \%$, respectively. Thus, it was found that PTXloaded F3, F6 and F9 tablets formulation were safe in normal cells but significantly toxic to immortalized cells represented as cancer cells for this investigation (Fig. 3).

\section{Conclusions}

In this study 27 PTX-loaded Micro protransfersome powder formulations (referred as Micro formulations based on transfersomes vesicles size post hydration) were prepared using one phospholipid, three surfactants and three lipid phase to carrier ratios to manufacture PTXloaded protransfersome tablets. LMH-based Micro protransfersome formulations F3, F6 and F9 were selected on the basis of excellent flowability via angle of repose, and good compressibility index due to smaller and uniform size and shape of $\mathrm{LMH}$, and thus used for tablet manufacturing. The three selected Micro protransfersome formulations were hydrated and Nano sized via probe sonication. These formulations were then converted back into dry form via spray drying (SD) or freeze drying (FD). Both Nano SD and Nano FD powder formulations showed poor to no flowability via AOR. Nano SD powder formulations demonstrated poor flowability via angle of repose, and were included for tablet manufacture. However, with no powder flowability exhibited by the Nano FD formulations, these were eliminated. Amongst the Micro and Nano protransfersome tablets, Micro tablets passed all the quality control tests (i.e. uniformity of weight, crushing strength, thickness and disintegration time), whilst Nano SD tablets failed the weight uniformity test and showed lower and inconsistent crushing strength and tablet thickness. Consequently, Nano formulations were excluded for further studies using medical nebulizers. The Ultrasonic nebulizer was found to be efficient in terms of performance for Micro protransfersome tablets as compared to Vibrating mesh nebulizer. Cytotoxicity studies exhibited that protransfersome tablet formulations F3, F6 and F9 (10\%) 


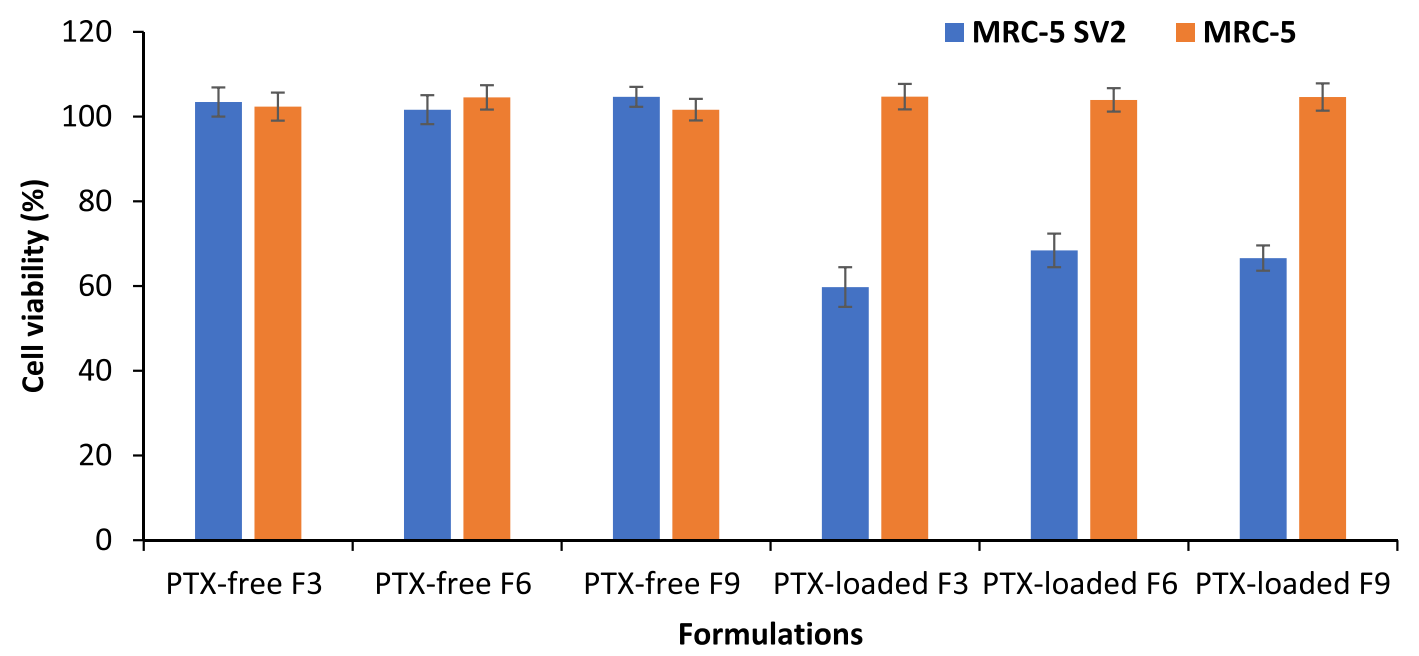

Fig. 3. Viability of MRC-5 and MRC-5 SV2 cell lines tested with transfersome generated from protransfersome tablet formulations F3, F6 and F9 using both PTX-free and PTX-loaded in black, flat-bottomed 96-well plates. Data are mean \pm SD, $n=4$.

were completely safe in normal cell lines while toxic to cancer cell lines. For future studies, these formulations can be further investigated in invitro using various lung cancer cells, as well as in-vivo study using florescent dye to detect their deposition in animal's lung by confocal microscopy. Furthermore, drug deposition in animal lung can be analysed via HPLC for quantification.

\section{CRediT authorship contribution statement}

Iftikhar Khan: Conceptualization, Supervision, Writing - original draft. Maria Apostolou: Data curation, Methodology. Ruba Bnyan: Software, Validation. Chahinez Houacine: Resources, Data curation. Abdelbary Elhissi: Visualization, Software. Sakib S. Yousaf: Investigation, Writing - review \& editing.

\section{Declaration of Competing Interest}

The authors declare that they have no known competing financial interests or personal relationships that could have appeared to influence the work reported in this paper.

\section{Acknowledgment}

We are very thankful to Lipoid, Switzerland for their generous supply of phosphatidylcholine (SPC, Lipoid S-100).

\section{Funding}

This research did not receive any specific grant from funding agencies in the public, commercial, or not-for-profit sectors.

\section{References}

Aulton, M.E., Taylor, K.M.G., 2018a. Powder flow. In: Aulton, M.E., Taylor, K.M.G. (Eds.), Aulton's Pharmaceutics: The Design and Manufacture of Medicines, fifth ed. Elsevier Health Sciences, London, UK, pp. 189-200.

Aulton, M.E., Taylor, K.M.G., 2018b. Pulmonary drug delivery. In: Aulton, M.E., Taylor, K.M.G. (Eds.), Aulton's Pharmaceutics: The Design and Manufacture of Medicines, fifth ed. Elsevier Health Sciences, London, UK, pp, 653-670.

Aulton, M.E., Taylor, K.M.G., 2018c. Tablets and compaction. In: Aulton, M.E., Taylor, K.M.G. (Eds.), Aulton's Pharmaceutics: The Design and Manufacture of Medicines, fifth ed. Elsevier Health Sciences, London, UK, pp. 520-530.

Bhushan Rajendra, R., Nayan Ashok, G., 2017. Transfersomes and protransfersome: ultradeformable vesicular system. In: Raj, K.K., Anil, K.S., Rajesh-Kumar, K. (Eds.), Novel Approaches for Drug Delivery. IGI Global, Hershey, PA, USA.

Bnyan, R., Khan, I., Ehtezazi, T., Saleem, I., Gordon, S., O'Neill, F., Roberts, M., 2018. Surfactant effects on lipid-based vesicles properties. J. Pharm. Sci. 107, 1237-1246.

BP, 2016a. Disintegration. Disintegration of Tablets and Capsules. Stationary Office on behalf of MHRA, London, UK.

BP, 2016b. Powder Flow. Angle of Repose. Stationary Office on behalf of MHRA, London, UK.

BP, 2016c. Powder Flow. Bulk Density and Tapped Density of Powder. Stationary Office on behalf of MHRA, London, UK.

BP, 2016d. Uniformity of Weight (Mass). Stationary Office on behalf of MHRA, London, UK.

Carr, R., 1965. Evaluating flow properties of solids. Chem. Eng. 72, 163-168.

Clay, M., Newman, S., Pavia, D., Lennard-Jones, T., 1983. Assessment of jet nebulisers for lung aerosol therapy. Lancet 322, 592-594.

di Sotto, A., Paolicelli, P., Nardoni, M., Abete, L., Garzoli, S., di Giacomo, S., Mazzanti, G., Casadei, M.A., Petralito, S., 2018. SPC Liposomes as possible delivery systems for improving bioavailability of the natural sesquiterpene $\beta$-caryophyllene: lamellarity and drug-loading as key features for a rational drug delivery design. Pharmaceutics $10,274$.

Dolovich, M.B., Dhand, R., 2011. Aerosol drug delivery: developments in device design and clinical use. Lancet 377, 1032-1045.

Elhissi, A., Taylor, K.M.G., 2005. Delivery of liposomes generated from pro liposomes using air-jet, ultrasonic and vibrating-mesh nebulisers. J. Drug Del. Sci. Technol. 15, 261-265.

Elhissi, A.M., Karnam, K.K., Danesh-Azari, M.R., Gill, H.S., Taylor, K.M., 2006. Formulations generated from ethanol-based proliposomes for delivery via medical nebulizers. J. Pharm. Pharmacol. 58, 887-894.

Elhissi, A.M.A., Ahmed, W., McCarthy, D., Taylor, K.M.G., 2011. A study of size, microscopic morphology, and dispersion mechanism of structures generated on hydration of proliposomes. J. Dispersion Sci. Technol. 33, 1121-1126.

Elhissi, A.M.A., Faizi, M., Naji, W.F., Gill, H.S., Taylor, K.M.G., 2007. Physical stability and aerosol properties of liposomes delivered using an air-jet nebulizer and a novel micropump device with large mesh apertures. Int. J. Pharm. 334, 62-70.

EPA. 2013. Particulate matter [Online]. USA: Environmental Protection Agency. Available: http://www.epa.gov/airscience/air-particulatematter.htm [Accessed 10. 01.2013].

Gelderblom, H., Verweij, J., Nooter, K., Sparreboom, A., 2001. Cremophor EL: the drawbacks and advantages of vehicle selection for drug formulation. Eur. J. Cancer 37, 1590-1598.

Ghazanfari, T., Elhissi, A.M.A., Ding, Z., Taylor, K.M.G., 2007. The influence of fluid physicochemical properties on vibrating-mesh nebulization. Int. J. Pharm. 339, 103-111.

Han, S.-M., Baek, J.-S., Kim, M.-S., Hwang, S.-J., Cho, C.-W., 2018. Surface modification of paclitaxel-loaded liposomes using d- $\alpha$-tocopheryl polyethylene glycol 1000 succinate: enhanced cellular uptake and cytotoxicity in multidrug resistant breast cancer cells. Chem. Phys. Lipids 213, 39-47.

He, L., Wang, G.L., Zhang, Q., 2003. An alternative paclitaxel microemulsion formulation: hypersensitivity evaluation and pharmacokinetic profile. Int. J. Pharm. 250, 45-50.

Hua, Z.-Z., Li, B.-G., Liu, Z.-J., Sun, D.-W., 2003. Freeze-drying of liposomes with cryoprotectants and its effect on retention rate of encapsulated ftorafur and vitamin A. Drying Technol. 21, 1491-1505.

Huschtscha, L.I., Holliday, R., 1983. Limited and unlimited growth of SV40-transformed cells from human diploid MRC-5 fibroblasts. J. Cell Sci. 63, 77-99.

Imtiaz-Ul-Islam Langrish, T.A.G., 2009. Comparing the crystallization of sucrose and lactosein spray dryers. Food Bioprod. Process. 87, 87-95.

Khan, I., Yousaf, S., Alhnan, M.A., Ahmed, W., Elhissi, A., Jackson, M.J., 2016. Design characteristics of inhaler devices used for pulmonary delivery of medical aerosols. In: Ahmed, W., Jackson, M.J. (Eds.), Surgical Tools and Medical Devices. Springer International Publishing, Cham, pp. 573-591.

Khan, I., Yousaf, S., Subramanian, S., Alhnan, M.A., Ahmed, W., Elhissi, A., 2018. Proliposome powders for the generation of liposomes: the influence of carbohydrate carrier and separation conditions on crystallinity and entrapment of a model 
antiasthma steroid. AAPS PharmSciTech 19, 262-274.

Khan, I., Yousaf, S., Subramanian, S., Korale, O., Alhnan, M.A., Ahmed, W., Taylor, K.M.G., Elhissi, A., 2015. Proliposome powders prepared using a slurry method for the generation of beclometasone dipropionate liposomes. Int. J. Pharm. 496 342-350.

Lachman, L., Lieberman, H.A., Kanig, J.L., 1986. The Theory and Practice of Industrial Pharmacy. Lea Febiger, Philadelphia, pp. 296-300.

Lawaczeck, R., Kainosho, M., Chan, S.I., 1976. The formation and annealing of structural defects in lipid bilayer vesicles. Biochim. Biophys. Acta (BBA) - Nucleic Acids Protein Synth. 443, 313-330.

Lehtonen, J.Y., Kinnunen, P.K., 1994. Changes in the lipid dynamics of liposomal membranes induced by poly(ethylene glycol): free volume alterations revealed by interand intramolecular excimer-forming phospholipid analogs. Biophys. J. 66, 1981-1990.

Leung, K.K.M., Bridges, P.A., Taylor, K.M.G., 1996. The stability of liposomes to ultrasonic nebulisation. Int. J. Pharm. 145, 95-102.

Maa, Y.F., Costantino, H.R., Nguyen, P.A., Hsu, C.C., 1997. The effect of operating and formulation variables on the morphology of spray-dried protein particles. Pharm. Dev. Technol. 2, 213-223.

Maury, M., Murphy, K., Kumar, S., Shi, L., Lee, G., 2005. Effects of process variables on the powder yield of spray-dried trehalose on a laboratory spray-dryer. Eur. J. Pharm. Biopharm. 59, 565-573.

Mc Callion, O.N.M., Taylor, K.M.G., Thomas, M., Taylor, A.J., 1996. Nebulisation of monodisperse latex sphere suspensions in air jet and ultrasonic nebulisers. Int. J. Pharm. 133, 203-214.

McCallion, O.N.M., Taylor, K.M.G., Bridges, P.A., Thomas, M., Taylor, A.J., 1996. Jet nebulisers for pulmonary drug delivery. Int. J. Pharm. 130, 1-11.

McGuire, W.P., Rowinsky, E.K., Rosenshein, N.B., Grumbine, F.C., Ettinger, D.S., Armstrong, D.K., Donehower, R.C., 1989. Taxol: a unique antineoplastic agent with significant activity in advanced ovarian epithelial neoplasms. Ann. Intern. Med. 111, 273-279.

Mercer, T.T., 1981. Production of therapeutic aerosols; principles and techniques. Chest 80, 813-818.

Najlah, M., Jain, M., Wan, K.-W., Ahmed, W., Albed Alhnan, M., Phoenix, D.A., Taylor, K.M.G., Elhissi, A., 2018. Ethanol-based proliposome delivery systems of paclitaxel for in vitro application against brain cancer cells. J. Liposome Res. 28, 74-85.

Nguyen, T.L., Nguyen, T.H., Nguyen, D.H., 2017. Development and in vitro evaluation of liposomes using soy lecithin to encapsulate paclitaxel. Int. J. Biomater. 2017, 7.

O'Callaghan, C., Barry, P.W., 1997. The science of nebulised drug delivery. Thorax 52 (Suppl 2), S31-S44.
Orr, G.A., Verdier-Pinard, P., McDaid, H., Horwitz, S.B., 2003. Mechanisms of Taxol resistance related to microtubules. Oncogene 22, 7280-7295.

Paolino, D., Celia, C., Trapasso, E., Cilurzo, F., Fresta, M., 2012. Paclitaxel-loaded ethosomes(R): potential treatment of squamous cell carcinoma, a malignant transformation of actinic keratoses. Eur. J. Pharm. Biopharm. 81, 102-112.

Prescott, J.K., Barnum, R.A., 2000. On powder flowability. Pharm. Technol. 20, 60-84. Rane, S., Prabhakar, B., 2009. Influence of liposome composition on paclitaxel entrapment and pH sensitivity of liposomes. Int. J. PharmTech Res. 1, 914-917.

Rathananand, M., Kumar, D., Shirwaikar, A., Kumar, R., Sampath Kumar, D., Prasad, R., 2007. Preparation of mucoadhesive microspheres for nasal delivery by spray drying. Indian J. Pharm. Sci. 69, 651-657.

Spencer, C.M., Faulds, D., 1994. Paclitaxel. A review of its pharmacodynamic and pharmacokinetic properties and therapeutic potential in the treatment of cancer. Drugs 48, 794-847.

Steckel, H., Eskandar, F., 2003. Factors affecting aerosol performance during nebulization with jet and ultrasonic nebulizers. Eur. J. Pharm. Sci. 19, 443-455.

Subramanian, S., Khan, I., Korale, O., Alhnan, M.A., Ahmed, W., Najlah, M., Taylor, K.M.G., Elhissi, A., 2016. A simple approach to predict the stability of phospholipid vesicles to nebulization without performing aerosolization studies. Int. J. Pharm. 502, $18-27$.

Taylor, K.M.G., McCallion, O.N.M., 1997. Ultrasonic nebulisers for pulmonary drug delivery. Int. J. Pharm. 153, 93-104.

Taylor, K.M.G., Morris, R.M., 1995. Thermal analysis of phase transition behaviour in liposomes. Thermochim. Acta 248, 289-301.

USP, 2015. Powder Flow. Angle of Repose, Compressability Index and Hausner Ratio. United Book Press, Baltimore, USA.

Vecellio, L., Abdelrahim, M.E., Montharu, J., Galle, J., Diot, P., Dubus, J.-C., 2011. Disposable versus reusable jet nebulizers for cystic fibrosis treatment with tobramycin. J. Cyst. Fibros. 10, 86-92.

Yang, D., Louden, C., Reinhold, D.S., Kohler, S.K., Maher, V.M., McCormick, J.J., 1992. In: Proceedings of the National Academy of Sciences, pp. 2237-2241.

Yang, S., Liu, C., Liu, W., Yu, H., Zheng, H., Zhou, W., Hu, Y., 2013. Preparation and Characterization of Nanoliposomes entrapping medium-chain fatty acids and vitamin C by lyophilization. Int. J. Mol. Sci. 14, 19763-19773.

You, Y.-Y., Suo, X.-B., Yue, J.-J., Xu, X., Zhang, H., 2017. Determination of entrapment efficiency of liposomal paclitaxel by RP-HPLC\&\#8727. Chinese J. Pharm. Anal. 37, $535-539$.

Zhu, H., Gooderham, N., 2002. Neoplastic transformation of human lung fibroblast MRC 5 SV2 cells induced by benzo[a]pyrene and confluence culture. Cancer Res. 62, 4605-4609. 